\title{
Aproximação do estudante de medicina à saúde da comunidade: um olhar atento na prevenção da automedicação
}

Approaching the medical student to the community health problems: A look closely at selfmedication prevention

Aproximación de los estudiantes de medicina a la salud comunitaria: una mirada más cercana a la prevención de la automedicación

\section{Resumo}

A precarização da assistência à saúde e dificuldades nos atendimentos de urgência afetam a sociedade, comprometendo a resolução imediata dos seus problemas de saúde. Sendo assim, quando existe doença, a população recorre a diversos meios para resolvê-la como a automedicação. O presente estudo objetivou discutir dados quantiqualitativos resultantes de um projeto de extensão envolvendo usuários de unidades de saúde e promover reflexões pedagógicas na formação profissional de estudantes de medicina. Trata-se de estudo transversal quanti-qualitativo delineado a partir de uma ação extensionista centrada na realização de rodas de conversa. Análises de frequências 
absoluta e relativa foram realizadas através do programa Jamovi ${ }^{\circledR}$. Os dados obtidos são resultantes das experiências das ações e informações coletadas nas rodas de conversa. Registrou-se que $73 \%$ dos usuários conhecem os riscos da automedicação, mas, ainda assim, essa prática é relatada por $89 \%$ deles. As principais justificativas para a automedicação foram: praticidade na aquisição $(72,3 \%)$, demora no atendimento ambulatorial $(33,2 \%)$ e difícil acesso aos profissionais de saúde (27,7\%). Observou-se também que as estratégias utilizadas permitiram que fossem promovidas reflexões sociais que tangenciaram os problemas da comunidade ao processo de ensino-aprendizagem dos estudantes, através de diálogos coletivos focados nas causas e consequências da automedicação, ocasionando maior aproximação entre os estudantes e a comunidade.

Palavras-chave: Automedicação; Promoção da saúde; Educação em saúde.

\begin{abstract}
Precariousness of health care, difficulties in emergency care affect society and prevent the immediate resolution of their health problems. Thus, when disease is present, the community resorts to different means to solve it, such as selfmedication practice. The present study aimed to discuss quantitative and qualitative data resulting from a community intervention project involving Healthcare Units users and to promote pedagogical reflections in medical students professional training. This is a quantitative-qualitative cross-sectional study idealized from an intervention project focused in the conversation wheels. Absolute and relative frequency analyses were performed by Jamovi ${ }^{\circledR}$ program. The data obtained as a result of experiences from the project and information collected in all conversation rounds. It was recorded that $73 \%$ of Healthcare Units users knowing the self-medication risks, but even so, this practice is reported by $89 \%$ of them. The main reasons for self-medications: convenience in acquisition (72.3\%), delay in outpatient care $(33.2 \%)$ and difficult access to health professionals $(27.7 \%)$. It was also observed that strategies used in the project made possible to promote social reflections through interactive dialogues focused on the causes and consequences of self-medication linking the community problems to the teaching-learning process of the students, resulting in a closer relationship between the students and community.
\end{abstract}

Keywords: Self-medication; Health promotion; Health education.

\title{
Resumen
}

La precariedad en la atención de la salud y las dificultades en la atención de urgencias afectan a la sociedad, comprometiendo la resolución inmediata de sus problemas de salud. Así, cuando existe una enfermedad, la población recurre a diferentes medios para solucionarla, como la automedicación. El presente estudio tuvo como objetivo discutir datos cuantitativos y cualitativos resultantes de un proyecto de extensión que involucra a usuarios de unidades de salud y promover reflexiones pedagógicas en la formación profesional de estudiantes de medicina. Se trata de un estudio transversal cuantitativo-cualitativo diseñado a partir de una acción extensionista centrada en la realización de ruedas de conversación. Los análisis de frecuencia absoluta y relativa se realizaron con el programa Jamovi ${ }^{\circledR}$. Los datos obtenidos son el resultado de las experiencias de acciones e información recopilada en los círculos de conversación. Se registró que el 73\% de los usuarios conocen los riesgos de la automedicación, sin embargo, esta práctica es relatada por el $89 \%$ de ellos. Los principales motivos de automedicación: conveniencia en la adquisición $(72,3 \%)$, retraso en la atención ambulatoria $(33,2 \%)$ y dificultad de acceso a los profesionales de la salud $(27,7 \%)$. También se observó que las estrategias utilizadas en el proyecto posibilitaron promover reflexiones sociales mediante diálogos interactivos enfocados en las causas y consecuencias de la automedicación vinculando los problemas de la comunidad al proceso de enseñanza-aprendizaje de los estudiantes, resultando en una relación más estrecha entre los estudiantes y la comunidad.

Palabras clave: Automedicación; Promoción de la salud; Educación en salud.

\section{Introdução}

O cenário epidemiológico atual da população brasileira expõe um aumento incisivo de doenças crônicas que exigem medicações de uso contínuo (Arrais et al., 2016), associado a um número expressivo de agravos ou comorbidades que requerem investigação e supervisão periódica da dosagem dos medicamentos prescritos (WHO, 2020; Lima et al., 2017). É nesse contexto que diversos indivíduos acabam realizando a chamada automedicação, seja para amenizar o sofrimento ou obter imediata resolutividade dos sinais e sintomas apresentados, o que acontece pelo uso abusivo de medicamentos que já foram prescritos ou através de prescrições antigas e, até mesmo, utilização sem prescrição (Silva et al., 2019).

Observa-se que a rotina no uso de medicamentos sem prescrição pode ser ocasionada pela falta de viabilidade imediata de um atendimento clínico, predispondo à utilização de receituários antigos e de medicamentos já conhecidos, reforçado pela facilidade na dispensação de algumas classes medicamentosas (Cortez et al., 2019). No entanto, essa prática pode ocasionar diversos efeitos adversos, sendo considerada, portanto, um problema de saúde pública. Na população brasileira, 
a automedicação tem crescido exponencialmente, o que é preocupante pelo fato de ocasionar graves consequências à saúde, seja em curto ou longo prazo (Oliveira et al., 2021).

A Organização Mundial da Saúde (OMS) registra que 50\% de todos os medicamentos são prescritos, dispensados ou usados de maneira incorreta. Esse elevado índice predispõe ao aumento dos riscos aos pacientes e contribui para o atraso no diagnóstico pelo mascaramento dos sintomas; além de resultar possíveis reações alérgicas e intoxicações que podem ser graves o suficiente para conduzir ao óbito (WHO, 2020). Somando-se a isso, o Sistema Nacional de Informações TóxicoFarmacológicas (SINITOX) em 2016, registrou mais de 95 mil casos de intoxicações em todo o país com 33,17\% destes devido ao uso inadequado de medicamentos (Brasil, 2016).

Nesse sentido, a população deve ser assistida não apenas do ponto de vista terapêutico, mas também no tocante à promoção de ações educativas e de prevenção que permitam o diálogo e produzam reflexões, sejam estas, individuais ou coletivas. Além disso, a reflexão sobre os problemas atuais vivenciados pela comunidade deve tangenciar o processo de ensino-aprendizagem dos estudantes da área de saúde, bem como, direcionar as suas ações para a resolução ou minimização das questões encontradas. Observa-se que a inserção do estudante ainda nos períodos iniciais do curso na comunidade que o cerca, possibilita que esses se tornem sujeitos da ação educativa, o que caracteriza uma aproximação efetiva junto à população assistida (Brito-Silva et al., 2019).

Pensando nisso, foi realizado um projeto de extensão intitulado "Automedicação: uma prática a ser combatida" com o intuito de conhecer o universo de questões que cercavam a comunidade vicinal à UFAL sobre automedicação e produzir reflexões sobre o tema; como também, articular ações de promoção à saúde que permitissem uma aproximação efetiva à comunidade, buscando despertar nos estudantes extensionistas a capacidade crítica e a compreensão das variáveis sociais que influenciavam tal prática.

Dessa forma, o objetivo do presente estudo, alicerçado nas ações realizadas, é apresentar e discutir dados quantiqualitativos resultantes de um projeto de extensão comunitária; além, de promover reflexões pedagógicas na formação profissional que permitam maior aproximação do estudante de medicina à saúde da comunidade.

\section{Metodologia}

Trata-se de um estudo transversal abordando aspectos quantitativos e qualitativos, delineado a partir de uma ação extensionista centrada na realização de rodas de conversas e abordagens educacionais sobre o tema automedicação em que todos os participantes possuíam livremente direito à fala. As ações ocorreram de abril de 2018 a setembro de 2019, envolvendo usuários de unidades de saúde localizadas em bairros periféricos da cidade de Maceió próximos à Universidade Federal de Alagoas (UFAL) e estudantes do curso de medicina com vistas à compreensão prática de concepções, conceitos e ações preventivas sobre os riscos da automedicação.

O Projeto de Extensão foi desenvolvido em quatro etapas, definidas como: 1. Exposição da importância do projeto junto às unidades de saúde, campo de prática dos estudantes de medicina da UFAL; 2. Sondagem e delineamento do perfil comunitário em cada unidade; 3. Pactuação das ações com as unidades de saúde; 4. Roda de conversa com os pacientes, aleatoriamente, presentes nas unidades de saúde nos dias em que as ações estavam programadas; entrega de cartilhas e apresentação de vídeos temáticos.

Registra-se que o desenvolvimento do projeto de extensão seguiu os direcionamentos exigidos pela Pró-Reitoria de Extensão Universitária (PROEXT/UFAL) e foi aprovado pelo Edital № 04/2018 PROCCAEXT/2018, registrado sob o número PJ166-2018. Além disso, após a realização da primeira etapa, as unidades de saúde autorizaram por meio de um termo de anuência as ações delineadas pelo projeto. Os usuários dos postos de saúde eram convidados a ouvir sobre os riscos da automedicação e a participar espontaneamente de uma roda de conversa sobre o tema com duração de 15 minutos, sem que 
nenhuma informação pessoal fosse registrada.

Enquanto os usuários aguardavam atendimento na recepção, cerca de 10 minutos antes das rodas de conversa, era distribuída uma lista a qualquer pessoa maior de 18 anos que se dispusesse a responde-la, com perguntas objetivas simples a respeito de possíveis hábitos de automedicação. Após a sondagem das respostas, era conversado com o grupo de usuários sobre os riscos apresentados pelo uso das classes de medicamentos mais citadas na lista de perguntas e, em tom de diálogo, eram realizadas ações educativas na tentativa de produzir reflexões efetivas sobre o tema.

Durante o momento da roda de conversa também era promovida a sensibilização e a reeducação quanto à automedicação praticada pela população; ressaltando a importância de multiplicar as informações recebidas. Além disso, era estimulada a consulta com o profissional capacitado, pois só este poderia realizar adequada prescrição medicamentosa.

Os usuários também recebiam uma cartilha autoexplicativa mostrando os riscos de se automedicarem. Ademais, através de um de sistema multimídia montado na recepção das unidades, foram exibidos pequenos vídeos socioeducativos de 4 minutos, gravados pelos estudantes de maneira teatralizada, em linguagem popular a respeito dos riscos do uso de medicamentos para grupos de risco específicos: idosos, gestantes e crianças, respeitando-se o fluxo normal de pacientes atendidos nas unidades.

Os dados obtidos são resultantes das experiências das ações e das informações colhidas imediatamente antes das rodas de conversa. As análises de frequências absoluta e relativa foram realizadas através do programa Jamovi®, versão 2.2.5.

\section{Resultados e Discussão}

As ações realizadas alcançaram um total de 500 usuários em duas unidades básicas de saúde do município de Maceió, Alagoas. As perguntas objetivas para a roda de conversa foram respondidas por 303 usuários; o que ficou dentro do esperado pelo cálculo do tamanho da amostra (297 indivíduos, IC 95\%), realizado através do programa OpenEpi ${ }^{\circledR}$.

Durante o delineamento do perfil comunitário (etapa 2 do projeto de extensão) e roda de conversas com os pacientes das unidades básicas de saúde (etapa 4), foi observado que a grande maioria, cerca de 73,4\% (Tabela 1), conheciam os riscos de se automedicarem e, ainda assim, $89 \%$ já a fez ou a fazia com frequência (Tabela 1).

Essa questão deve ser contextualizada como um problema de saúde pública; não à toa, Andrade et al. (2020) registraram que 52,8\% dos casos de intoxicação notificados de 2010 a 2017 foi por uso indevido de medicamentos. E nesta mesma linha de investigação, Secoli et al. (2018) relataram que as intoxicações por fármacos são responsáveis por 29\% das mortes por intoxicação no Brasil, sendo a maioria dos casos decorrente da automedicação.

Quando conversado com os usuários a respeito de temas que levavam ao processo da automedicação, foram levantadas questões como a facilidade em adquirir o produto e a dificuldade que muitos apresentam em ter acesso aos serviços de saúde, principalmente a população mais carente.

Nesse quesito, a praticidade na aquisição $(72,3 \%)$ e a grande facilidade no ato da compra $(51,3 \%)$ mostraram ser os principais fatores facilitadores na prática da automedicação (Tabela 1), estando consonantes com o estudo de Oliveira et al. (2018) o qual afirma que a facilidade de acesso às farmácias e drogarias predominam entre as justificativas para uso de medicamentos por conta própria. Segundo Silva (2020), atualmente no país há três vezes mais drogarias e farmácias que o recomendado pela OMS, sustentando o fato da praticidade na aquisição dos medicamentos. Além disso, de acordo com Pinto (2014) o marketing da indústria farmacêutica possui grande poder de indução a tais práticas, cujo investimento em campanhas pode ultrapassar os gastos em atividades de pesquisa e desenvolvimento. Isto revela que, além do possível desconhecimento dos efeitos das drogas, há também uma forte influência advinda da estratégia de comercialização dos medicamentos (Pinto, 2014).

Oliveira et al. (2018) ressaltam que a automedicação é prevalente entre os brasileiros devido à precariedade da 
atenção básica e que o sistema público de saúde deveria fornecer a todos o acesso aos serviços sem que houvesse a necessidade de longos períodos aguardando um atendimento médico. A Tabela 1 mostra que 33,2\% alegaram demora no atendimento ambulatorial e $27,7 \%$ difícil acesso aos profissionais da saúde. Dessa forma, torna-se tentadora a busca diretamente às farmácias para a aquisição imediata dos medicamentos quando comparada à espera por uma consulta médica. Além disso, é comum que aqueles que conseguiram consultas e receituários, repassem informações e indiquem medicações a amigos e familiares, o que foi reportado por 46,9\% dos indivíduos, Tabela 1.

Tabela1: Coleta de informações a respeito do motivo da automedicação.

\begin{tabular}{lcc}
\hline Variáveis & $\mathbf{n}$ & $\mathbf{\%}$ \\
\hline 1. Já fez uso de medicação sem prescrição? & $(\mathbf{3 0 3})$ & \\
Sim & 271 & 89,4 \\
Não & 32 & 10,6 \\
2. Sabe que a automedicação pode trazer riscos à saúde? & 199 & 73,4 \\
Sim & 72 & 26,6 \\
Não & & \\
3. Já indicou algum medicamento a alguém? & 127 & 46,9 \\
Sim & 144 & 53,1 \\
Não & & \\
4. Qual a frequência de consultas com o médico e de check-ups? & 71 & 26,2 \\
Raramente & 91 & 33,6 \\
1 vez ao ano & 109 & 40,2 \\
Mais de 1 vez ao ano & & \\
5. Já fez compra de medicamentos baseada em prescrições antigas? & 181 & 66,8 \\
Sim & 90 & 33,2 \\
Não & & \\
6. Com qual frequência ler a bula das medicações? & 80 & 29,5 \\
Nunca & 48 & 17,7 \\
Raramente & 143 & 52,8 \\
Frequentemente/sempre & & \\
7. Motivo que o(a) influenciou a fazer a automedicação & 139 & 51,3 \\
Facilidade de compra & 196 & 72,3 \\
Praticidade & 90 & 33,2 \\
Demora no atendimento ambulatorial & 75 & 27,7 \\
Difícil acesso aos profissionais & 26 & 9,6 \\
Falta de qualidade no atendimento & &
\end{tabular}

Fonte: Autores (2022).

Dessa forma, a tarefa de realinhar hábitos advindos da comunidade e do ambiente familiar não é algo pontual, mas uma atividade continuada e multidisciplinar. Em um ambiente como a unidade de saúde, esse processo pode ser facilitado, haja vista a discussão de interesses coletivos e que podem ser trabalhados através de ações dinâmicas com formação construtiva. Esse tipo de abordagem além de permitir o relato espontâneo de experiência, promove acolhimento, desenvolvimento de pensamento crítico sobre o tema e, por vezes, forma multiplicadores da informação (Melo, 2019).

Essas questões, diretamente ligadas a situações sociais associadas à prática da profissão, permitem que sejam trabalhadas nos estudantes de medicina o processo de empatia, permitindo que vários temas fossem levantados como medo, ansiedade, angústia e adoecimento.

Nesse sentido, quando perguntado sobre frequência e rotina de consultas médicas, mais de $70 \%$ responderam realizar consultas/check-ups ao menos $1 \mathrm{vez}$ ao ano, semelhante a outros estudos realizados na região sul do Brasil, como em Santa Catarina, apontando-se uma prevalência de consultas médicas por ano igual a 76\% de 2022 adultos (Boing et al., 2010) e no Rio Grande do Sul, registrando cerca de 72\% (Dias-da-Costa et al., 2008). Contudo, observa-se que esse cenário não representa a realidade daquelas pessoas que necessitam de uma avaliação imediata ou esporádica, mas sim daqueles pacientes com doenças crônicas, haja vista a necessidade que os mesmos apresentam de acompanhamento contínuo e, portanto, de 
manutenção de agendamentos programados periodicamente.

Ressalta-se que dentre os fatores que facilitam a prática da automedicação também figura o uso de prescrições antigas de medicamentos que não são de uso contínuo. Segundo Araújo (2014), esse comportamento se faz mais presente entre os indivíduos mais idosos e também por aqueles responsáveis por crianças, tendo em vista que nessas faixas etárias as afecções que levam ao consumo de medicamentos já prescritos são mais prevalentes.

Dessa forma, percebe-se que quando se abre um espaço para a promoção da saúde junto à comunidade é importante que as ações sejam voltadas a todos os grupos identificados e que as estratégias sejam organizadas de maneira a alcançar o maior número de pessoas. Portanto, a identificação de um público formado por idosos, gestantes e mulheres permitiu que diferentes estratégias fossem utilizadas para aproximar os estudantes aos diferentes usuários.

Foi observado que $52,8 \%$ dos participantes da roda de conversa costumam fazer a leitura da bula dos medicamentos com frequência. E mesmo consultando os possíveis efeitos colaterais e riscos da administração do fármaco, a grande maioria afirmou fazer a automedicação rotineiramente sem assistência de um profissional da saúde, o que pode estar relacionado à falta de entendimento dos pacientes sobre os termos técnicos da bula. Segundo Paula et al. (2009), mais de 80\% dos pacientes leem as bulas e dentre as principais dificuldades, destacam-se: a linguagem complexa e o tamanho reduzido das letras que inviabilizam a completa compreensão do texto.

Esses aspectos permitem aos estudantes perceberem que nem todas as arestas na história clínica do paciente podem ser facilmente resolvidas, e muito menos, tentar resolvê-las apenas do ponto de vista clínico e farmacológico. Deve-se imergir nos aspectos sociais que também contribuíram com o processo de adoecimento e que levaram à tomada de decisões imediatas para a resolução do problema (Brito-Silva et al., 2019).

Dados da presente ação mostram que 44,3\% dos participantes fizeram uso da automedicação por apenas um dia e 20,7\% por até dois dias (Tabela 2). Informações semelhantes foram encontradas por Amaral (2019), em que a maioria dos indivíduos (51\%) referiu que a duração do uso dos medicamentos variava entre 1 e 2 dias. Além disso, o mesmo estudo também enfatizou que os principais grupos de medicamentos utilizados foram os analgésicos $(56,9 \%)$, os anti-inflamatórios $(43,1 \%)$ e os antipiréticos $(18,8 \%)$; o que corrobora os achados coletados nas rodas de conversa, em que anti-inflamatórios, analgésicos e antipiréticos foram citados por $92,6 \%$ das pessoas (Tabela 2).

Ressalta-se que o uso excessivo dessas classes medicamentosas pode trazer diversos efeitos colaterais, alguns mais brandos como cefaleia de rebote ocasionada por analgésicos com quantidades significativas de cafeína; outros mais graves como nefrite intersticial, reações alérgicas e, até mesmo, hepatotoxicidade e hemorragia gastrintestinal decorrentes principalmente do abuso de AINEs (anti-inflamatórios não esteroides) como ibuprofeno e naproxeno (Oliveira et al., 2018).

Dessa forma é necessário entender que os riscos podem sobrepujar os benefícios e resultar em agravos com diferentes intensidades de gravidade, podendo chegar até ao óbito. Esse é um ponto crítico da discussão em uma roda de conversa, pois a confiança no que está sendo relatado, como também no que está sendo transmitido pelos estudantes permite o estabelecimento da conscientização. 
Tabela 2: Coleta de informações sobre causa-consequência da automedicação.

\begin{tabular}{lcc}
\hline Variáveis & $\mathbf{n}$ & $\mathbf{\%}$ \\
\hline 8. O que sentia quando usou da última vez medicamento sem prescrição? & $(\mathbf{3 0 3})$ & 45,0 \\
Tosse/gripe & 122 & 59,4 \\
Inflamação & 161 & 52,02 \\
Dor muscular/ossos & 141 & 70,5 \\
Dor de cabeça & 191 & 16,2 \\
Sintomas estomacais ou intestinais & 44 \\
Problemas de pele & 14 & 5,2 \\
Problemas circulatórios & 13 & 4,8 \\
Febre & 68 & 25,1 \\
Alergia & 40 & 14,8 \\
9. Por quanto tempo usou a medicação? & 120 \\
Até dia & 56 \\
Até 2 dias & 95 \\
Acima de 2 dias & 44,3 \\
10. Ocorreu o surgimento de algum sinal ou sintoma após a automedicação? & 20,7 \\
Mal estar & 35,1 \\
Sonolência & 19 \\
Dores estomacais & 21 \\
11. Classe de medicamentos mais citada & 13 \\
Anti-inflamatórios, analgésicos e antitérmicos & & 7,0 \\
Antibióticos & 251 \\
Antieméticos & 3,7 \\
Antialérgicos & 39 \\
Anticoncepcionais & 22 \\
12. Utiliza medicação de uso contínuo? & 25 \\
Sim Não & 11 \\
\hline
\end{tabular}

Fonte: Autores (2022).

A Tabela 2 também aborda os sintomas que levaram os usuários a fazerem uso de medicamentos não prescritos, sendo eles, principalmente: dor de cabeça (70,5\%), inflamação $(59,4 \%)$, dor muscular/ossos $(52,02 \%)$ e tosse/gripe $(45,0 \%)$.

Nesse contexto, um estudo nacional destacou entre os relatos para a automedicação: dor de cabeça/febre, resfriado/gripe, dores musculares e cólica (Matos et al., 2018). Dados semelhantes também foram encontrados em estudo internacional realizado com a população de Portugal que registrou os principais sinais e sintomas que levaram ao uso de medicamentos por conta própria: dores, gripe, constipação, febre, infecções e tosse (Amaral et al., 2019).

Pode ser entendido, nesse sentido, que os dados encontrados na presente ação estão em consonância com estudos recentes, sendo a dor o principal sintoma encontrado. Isso permite registrar a importância da aproximação dos estudantes ao tema e à comunidade, o que reflete na relevância das medidas de intervenção utilizadas na ação (etapa 4).

Portanto, no que diz respeito às cartilhas e aos vídeos distribuídos/transmitidos durante a ação, observou-se que foram alcançados cerca de 500 usuários que estavam presentes nas intervenções. A Figura 1 mostra momentos da exibição dos vídeos, entrega das cartilhas e chamamento para a participação ativa nas rodas de conversa. 
Figura 1: Imagens dos estudantes convidando os usuários para as rodas de conversa, distribuição das cartilhas e exibição dos vídeos de curta duração abordando os riscos da automedicação.
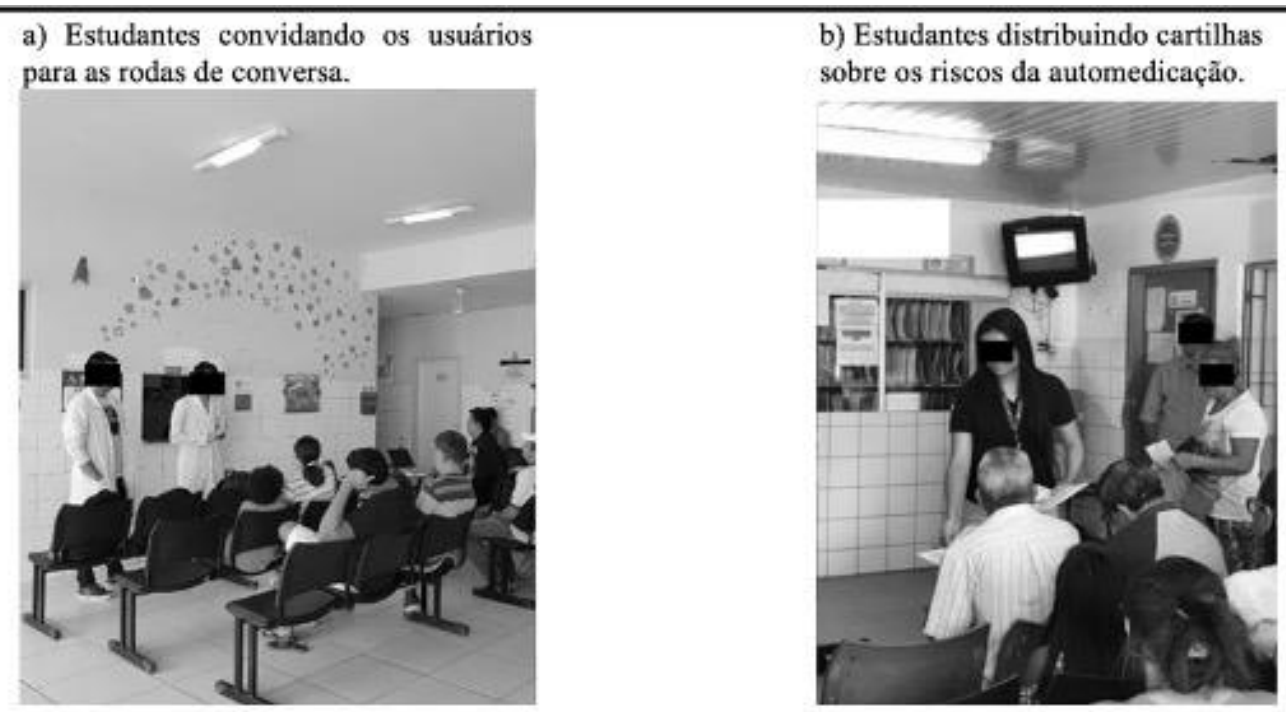

c) Estudantes exibindo os videos de curta duração abordando os riscos da automedicação.

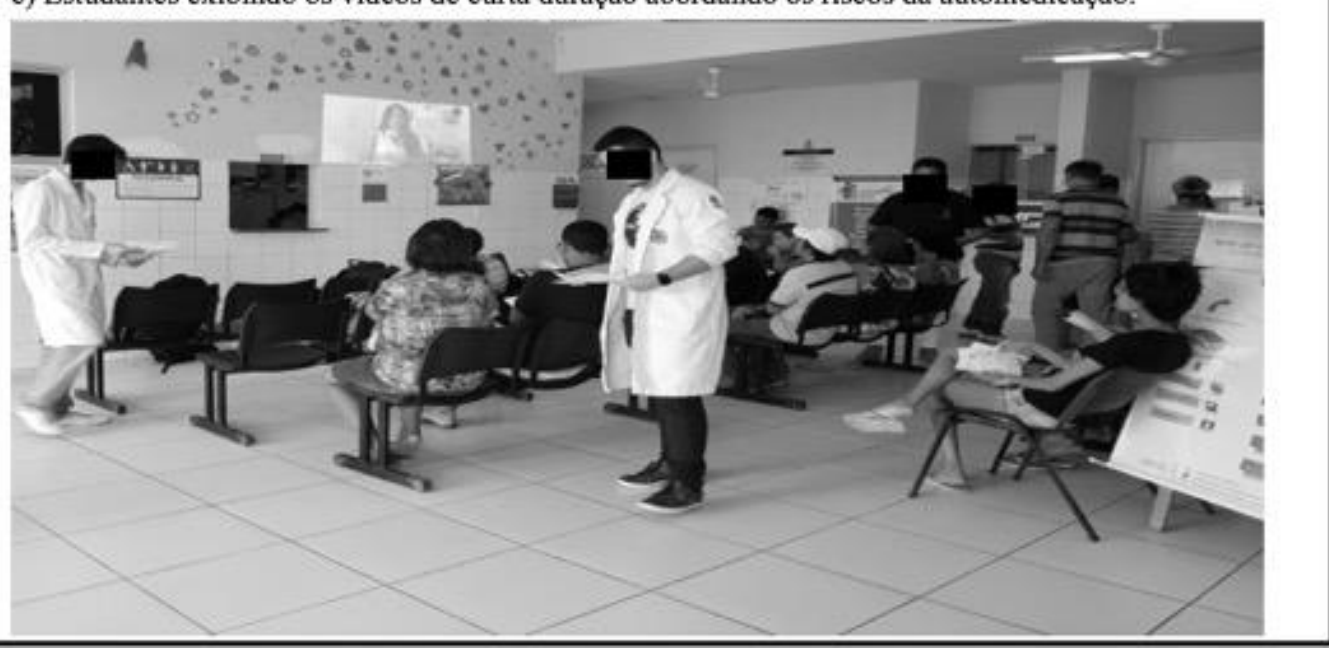

Fonte: Autores (2022).

Em todos os momentos foi preconizado o uso de uma linguagem simples e objetiva, permitindo que todos possuíssem o direito ao momento de fala, tornando a comunicação acessível e multidirecional, o que pode ser identificada também na linguagem empregada nas cartilhas, conforme apresentada na Figura 2. 
Figura 2: Cartilha autoexplicativa sobre os riscos da automedicação.

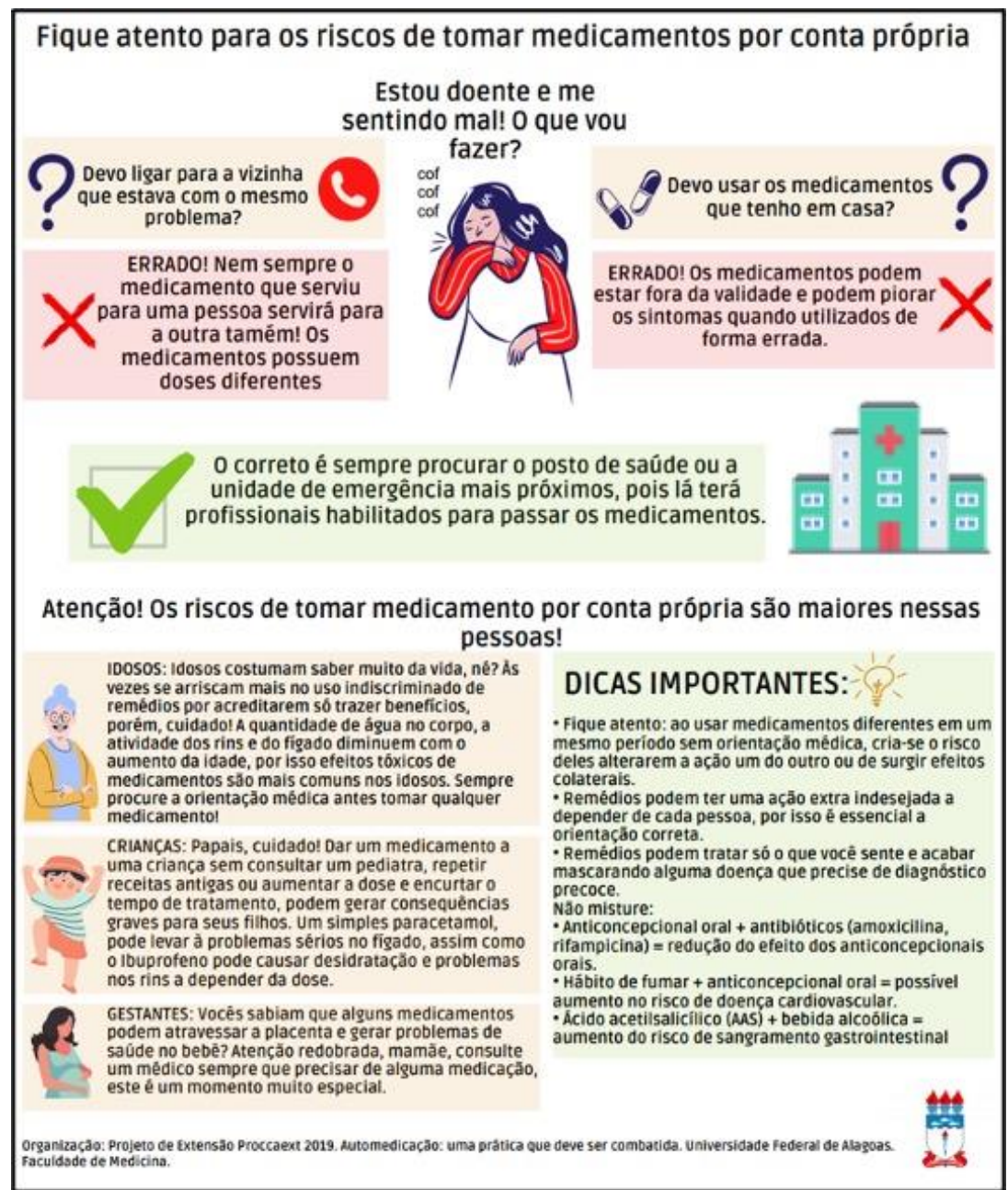

Fonte: Autores (2022). Imagens disponibilizadas livremente na internet.

Sempre após a exposição dos vídeos, havia um momento para que fossem sanadas as dúvidas postas pelos expectadores. A importância de dar voz, ou seja, não apenas falar, mas também ouvir permite que todos possuam participação ativa espontânea e detenham maior interesse nos temas expostos. Essas ações dialogadas, interativas e de aproximação aos temas relevantes da comunidade permitiram maior aproximação do estudante de medicina à saúde da comunidade em busca de um olhar atento na prevenção da automedicação.

Todas as rodas de conversa permitiram trazer elementos essenciais e diferenciais que estimularam espontaneamente o processo de reflexão, com o intuito de influenciar em tomadas de decisões futuras a respeito do uso de medicações por conta própria. Esses elementos podem ser descritos como fatores de empatia, caracterizados pelos gestos, pela colocação das palavras, pelo tom da fala e ideias, assim como pela reciprocidade do olhar (Melo, 2019). Além disso, a exposição de exemplos palpáveis e inseridos dentro do que é vivenciado pela comunidade permitiu maior aproximação com o público ouvinte, o que despertou o ensejo de fala, da comunicação e resultou, consequentemente, em troca de experiências.

Diante do exposto, o momento de espera em um ambiente ambulatorial, clínico ou hospitalar que, por vezes, traz ansiedade, pode ser substituído por práticas educativas que permitem além da informação, a produção de suporte no esclarecimento de dúvidas temáticas (Feitosa et al., 2019). Ainda, o diálogo estabelecido entre estudantes e usuários cria a oportunidade de aproximação efetiva entre estes, além de permitir que os futuros profissionais se tornem sujeitos da ação educativa junto a sua comunidade (Brito-Silva et al., 2019). 
Nesse sentido, entende-se que proporcionar reflexão a partir de uma ação dialogada requer a investigação da origem dos problemas levantados e não somente da sua exposição. A interligação das falas dos usuários às perguntas subjetivas coletadas aponta que o acesso ao profissional prescritor ainda representa o principal entrave a ser vencido quando se trata da utilização de medicamentos sem prescrição. Essa premissa abre espaço para que haja a interligação dos problemas encontrados com questões sociais, econômicas e políticas, especificamente políticas públicas (Secoli et al., 2018).

Adiciona-se a este contexto, o advento da pandemia da Covid-19 que evidenciou o uso acentuado de medicações propagadas com o propósito preventivo ou até mesmo curativo, mas que contrariamente, aumentaram o risco dos efeitos adversos e das intoxicações (PAHO, 2020). Dessa forma, percebe-se um déficit social, cultural e de políticas públicas educacionais voltadas para o uso abusivo de medicamentos e sem prescrição. A análise do contexto epidemiológico atual demonstra que a sociedade ainda carece de práticas educativas robustas e que alcancem todos os estratos sociais, haja vista que independe do nível de escolaridade (Melo et al., 2021).

É nesse cenário reflexivo que o estudante também toma ciência de que sua formação deve ser não apenas intelectualcognitiva, mas, para além disso, é necessário um olhar atento com responsabilidade social para que as iniciativas no processo de reeducação, promoção e prevenção à saúde sejam eficientes (Brito-Silva et al., 2019). Dessa forma, mais que compreender a temática, a abordagem educativa e preventiva deve refletir o cenário social e educacional da comunidade, bem como as políticas públicas voltadas à população.

\section{Considerações Finais}

Observou-se que a grande maioria dos usuários das unidades de saúde conhecia os riscos da automedicação e, ainda assim, a fazia com frequência em decorrência da dificuldade no acesso às consultas ambulatoriais e da facilidade na aquisição das medicações sem a prescrição. Além disso, os principais sintomas relatados para o uso de medicamentos por conta própria foram dores de cabeça e muscular, inflamação e tosse, juntamente com relatos da utilização das classes medicamentosas mais citadas como analgésicos e anti-inflamatórios, administrados em sua maioria por um prazo de até dois dias.

No campo reflexivo, as abordagens educativas contribuíram para a sensibilização do público-alvo, despertando-se multiplicadores da informação, haja vista que a equipe recebeu convites para fazer as mesmas programações em escolas, centros comunitários e em reuniões religiosas. Foram estimulados o devido descarte de todos os medicamentos fora da validade e a ida às consultas e reavaliações, ainda que com datas distantes; como também uma maior frequência de consultas com a equipe multidisciplinar.

Outros importantes ganhos decorrentes das ações extensionistas também foram identificados nos estudantes, pois tais ações permitiram aproximá-los das necessidades da comunidade e refletir sobre importantes questões sociais que se somam ao saber científico, criando-se uma relação translacional de retorno à prática social que os tornarão profissionais mais humanizados.

Portanto, observa-se que as abordagens práticas na sensibilização de temas importantes que afetam a saúde da comunidade devem ser realizadas de forma rotineira para que haja, de fato, a consolidação de novos hábitos e jamais deve ser encarado como falta de interesse pela comunidade em absorver as mudanças propostas, uma vez que o binômio causa-efeito deve ser modificado conjuntamente.

\section{Referências}

Amaral, O., Veiga, N., Nelas, P., Coutinho, E., \& Chaves, C. (2019). Automedicação na comunidade: um problema de saúde pública. International Journal of Developmental and Educational Psychology. Revista INFAD de Psicología, 3(1), 423-432. 
Araújo, A. P. (2014). Estudos brasileiros sobre automedicação: uma análise da literatura. Monografia (Bacharelado em Farmácia), Universidade de Brasília, Brasília, BSB, Brasil. https://bdm.unb.br/handle/10483/8734

Arrais, P. S. D., Fernandes, M. E. P., Dal Pizzol, T. S., Ramos, L. R., Mengue., S. S., Vera, L. L., Tavares, N. U. L., \& Farias, M. R. (2016). Prevalence of self-medication in Brazil and associated factors. Revista de Saúde Pública, 50 (supl 2:13s), 1s-11s.

Boing, A. F., Matos, I. B., Arruda, M. P., Oliveira, M. C., \& Njaine, K. (2010). Prevalência de consultas médicas e fatores associados: um estudo de base populacional no sul do Brasil. Revista da Associação Médica Brasileira, 56(1), 41-6.

Brasil. (2016). Casos, Óbitos e Letalidade de Intoxicação Humana. Recuperado 25 de novembro, 2021, de https://sinitox.icict.fiocruz.br/dados-nacionais.

Brito-Silva, A. L., Sousa, S. C., Chaves, A. C. F., Sousa, S. G. C., Andrade, T. M., \& Filho, D. R. R. (2019). Importância da Extensão Universitária na Formação Profissional: Projeto Canudos. Revista de Enfermagem UFPE on line, 13(e242189), 1-8.

Cortez, A. C. L., Silva, C. R. L., Silva, R. C. L., \& Dantas, H. M. (2019). Aspectos gerais sobre a transição demográfica e epidemiológica da população brasileira. Enfermagem Brasil, 18(5), 700-709.

Dias-da-Costa, J. S., Gigante, D. P., Horta, B. L., Barros, F. C., Victora, C. G. (2008). Utilização de serviços de saúde por adultos da coorte de nascimentos de 1982 a 2004-5, Pelotas, RS. Rev Saúde Pública. 42(Suppl 2), 51-9).

Feitosa, A. L. F., Silva, F. L., Santos, K. S. O. Silva, L. K. G., Rocha., M. C. G., \& Andrade, M. F. L. O. (2019). Sala de espera: estratégia de educação em saúde no contexto da atenção básica. Revista Brasileira de Educação e Saúde, 9(2), 67-70.

Lima, M. G., Álvares, J., Junior, A. A. G., Costa, E. A., Guibu, I. A., Soeiro, O. M., Leite, S. N., Karnikowski, M. G. O., Costa, K. S., \& Acurcio, F. A. (2017). Indicadores relacionados ao uso racional de medicamentos e seus fatores associados. Revista de Saúde Pública, 51(Supl 2:23s), 1s-9s.

Matos, J. F., Pena, D. A. C., Parreira, M. P., Santos, T. C., \& Coura-Vital, W. (2018). Prevalência, perfil e fatores associados à automedicação em adolescentes e servidores de uma escola pública profissionalizante. Cadernos Saúde Coletiva, 26 (1): 76-83.

Melo, E. S. (2019). Roda de Conversa como Estratégia para Gestão e Educação Permanente em Saúde. Revista Portal: Saúde E Sociedade, 4(2), $1152-1159$.

Melo, J. R. B., Duarte, E. C., Moraes, M. V., Fleck, K., \& Arrais, P. S. D. (2021). Automedicação e uso indiscriminado de medicamentos durante a pandemia da COVID-19. Cadernos de Saúde Pública, 37(4), 1-5.

Oliveira, J. V. L., Costa, F. B., Porfírio, V. N., Silva, M. M. M., Cunha, A. B. O. C., Silva, N. C., Nascimento, V. J. O. A., França, A. M. M., Melo, M. L. R. S., Silva, R. F. C., Costa, M. D. T., \& Filho, L. S. S. (2021). A automedicação no período de pandemia de COVID-19: Revisão integrativa. Research, Society and Development, 10(3), 1-11.

Oliveira, V. C., Cavalcante, M. G., Vasconcelos, L. F. \& Carvalho, A. M. R. (2018). Perfil da automedicação em uma farmácia comunitária no município de Itapipoca-CE. Revista Expressão Católica Saúde, 3(1), 64-70.

Pan American Health Organization. (2020). Ongoing living update of COVID-19 therapeutic options: summary of evidence. https://iris.paho.org/handle/10665.2/52719.

Paula, C. S., Costa, C. K., Miguel, M. D., Zanin, S. M. W., \& Spinillo, C. G. (2009). Análise crítica de bulas sob a perspectiva do usuário de medicamentos. Visão Acadêmica, 10(2), 123-133.

Pinto, C. A. S. (2014). A indústria farmacêutica da américa latina: um estudo comparativo. Dissertação de mestrado, Universidade Estadual Paulista, São Paulo, SP, Brasil. https://repositorio.unesp.br/handle/11449/115740.

Secoli, S. R., Marquesini, E. A., Fabretti, S. C., Corona, L. P., \& Romano-Lieber, N. S. (2018). Tendência da prática de automedicação entre idosos brasileiros entre 2006 e 2010: Estudo SABE. Revista Brasileira de Epidemiologia, 21(suppl2:e180007. supl.2), 1-18.

Silva, I. D. D., Bezerra, I. N., M., Pimenta, I. D. S. F., Silva, G., Wanderley, V. B., Nunes, V. M. A., Souza, D. L. B., \& Piuvezam, G. (2019). Acesso e implicações da automedicação em idosos na atenção primária à saúde. Journal Health NPEPS, 4(2), 132-150.

Silva, T. F. Q., Grillo, L. P., Lacerda, L. L. V., \& Mezadri, T. (2020). Prevalência de automedicação e características de acesso a anti-inflamatórios em adultos no município de Navegantes, Santa Catarina. Revista Ciências em Saúde, 10(2), 20-27.

World Health Organization. (2020). Noncommunicable diseases: Progress monitor 2020. World Health Organization, 2020. https://www.who.int/publications/i/item/9789240000490. 